\title{
Associating User's Psychology into Quality of Service: An Example of Web Adaptation Services
}

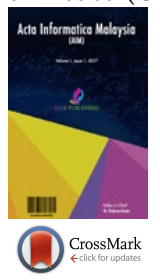

\section{Mohd Farhan Md Fudzee, Juliana Mohamed, Azizul Azhar Ramli, Shahreen Kasim, Hairulnizam Mahdin, Mohd Norasri Ismai, Azizan Ismail \& Mohd Hanif Jofri}

Faculty of Computer Science and Information Technology University Tun Hussein Onn Malaysia Parit Raja, 86400 Batu Pahat, Johor, Malaysia \{farhan,azizulr, shahreen, hairuln, norasri, mhanif\}@uthm.edu.my

This is an open access article distributed under the Creative Commons Attribution License, which permits unrestricted use, distribution, and reproduction in any medium, provided the original work is properly cited.

\section{ARTICLE DETAILS}

Article history:

Received 22 January 2017

Accepted 03 February 2017

Available online 05 February 2017

Index Terms:

Quality of service, Web adaptation service, User psychology

\begin{abstract}
- Content adaptation is a potential solution for tailoring multimedia web content according to the users' preferences and heterogeneous devices' constraints. Content adaptation can be done as third party service over the Internet. Users may pay for the service thus demand quality. The quality should include the human psychological factors. One of these factors is the maximum time a user can wait for the output to be displayed. Thus, response time is one of the qualities of service (QoS) to be considered in assessing the deliverability of content adaptation services. However, the advertised response time may not be deliverable accordingly during the actual service execution due to heavy load. Practically, the service provider should able to determine a current deliverable response time before the service level agreement (SLA) is settled with the users. In this paper, we propose a strategy for service providers to evaluate incoming requests and capable of offering the new response time. The proposed strategy takes into account the current server load and enables a mechanism for the user to evaluate whether the new response time can be accepted or not. We analyzed the performance of the proposed strategy in terms of SLA settlement under various conditions. The results indicate that the proposed strategy performs well.
\end{abstract}

\begin{abstract}
1. Introduction
Content adaptation has in fact already gained considerable importance in today's multimedia communications and will certainly become an essential functionality of any service, application or system in the near future (Anna Carreras et. al, 2009). As the heterogeneity of mobile device such as smart phone and tabs growth, searching websites through mobile device is also demanding. The recent trend is that content adaptations are largely demanded and became profitable mobile contents of its quality to the Internet as third party services (Arai \& Tolle, 2010). Existing content adaptation systems employing these approaches such as client-side, serverside or proxy-side adaptation (Malandrino et al., 2010) operate in isolation, often encounter limited adaptation functionality, get overload if too many concurrent users and open to single point of failure, thus limiting the scope and scale of their services (Yang \& Shao, 2007).
\end{abstract}

Digital multimedia services and devices are now mediated through increasingly proportion of human activities such as social interactions, entertainment, shopping, and gathering information (Kosinski, Stillwell \& Graepel, 2013). To get more accuracy and rapid information, users may pay for the service thus demand quality. Generally, the response of those services is not very immediately but rather delayed to a certain extent depends on the type of request and the type of desired response. At the end, these response times is largely dominated to user-perceived quality (Egger Hossfeld, Schatz, \& Fiedler, 2012).

The idea of establishing content adaptation as a service to allow potential solution for tailoring multimedia web content according to the users' preferences and heterogeneous devices' constraints has been endorsed Typically, there are many service providers offering a variety of content adaptation that can be suit. Therefore, the solution for these services is to cooperate with each other through service composition to completely serve the request that they cannot attain individually. This is termed as Serviceoriented Content Adaptation (SOCA). SOCA presents a model to enable content adaptation to be consumed as Web services (Fudzee \& Abawajy, 2011). However, current solutions from (Alhamad, Dillon \& Chang, 2010) do not take into account the human psychology in negotiating with services. Figure 1 shows the architecture of service level agreement (SLA) by SOCA framework. This system is based from the service oriented architecture (SOA). The purpose of the SOCA framework is to give out user expectation by offering added value of content and also to provide flexible and scalable service-based content delivery mechanism. The framework consists of components that provide access to content servers, develop user request to source format, manage and provide content description (Fudzee, Abawajy, \& Deris, 2010)

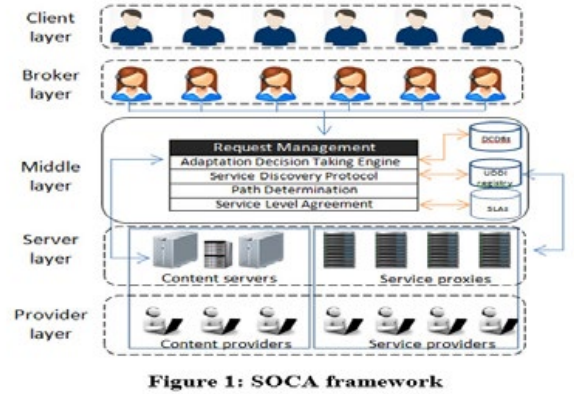

Commonly, SOCA enables an adapted content to be delivery by a number of services; service composition based on quality of service (QoS) is known to be a practical solution. Hence, response time is one of the qualities of service (QoS) to be considered in assessing the deliverability of content adaptation services. However, the advertised response time may not be deliverable accordingly during the actual service execution due to heavy load.

The quality of mobile services in content adaptation should include the human psychological factors. One of these factors is the maximum time a user can wait for the output to be displayed. Lobo, Kaskaloglu, Kim \& Herbert (2011) stress that there will be more frustration while making longer enough of time to wait for pages to load, so as a result they usually quit the task or try to find information to another site. This is also relevant to content adaptation service response time.

Practically, the service provider should able to determine a current deliverable response time before the service level agreement (SLA) is settled with the users. This strategy has been found out from (Fudzee, 2011) by proposed QoS negotiation modeling of response time by considering one to one negotiation strategy (from broker as user on behalf to service provider). In this paper, we propose a strategy for service providers to evaluate incoming requests and capable of offering the new response time. The proposed strategy takes into account the current server load and enables a mechanism for the user to evaluate whether the new response time can be accepted or not. We analyzed the performance of the proposed strategy in terms of SLA settlement under various conditions. The results indicate that the proposed strategy performs well.

This paper consist of six section which includes our related work in section 2 , the proposed solution in section 3 , the performance evaluation in 
section 4, result and discussion in section 5 and the conclusion for this paper in section 6 .

\section{Literature Review}

Previous studies have overcome the problems of service provider from SOCA framework. SOCA comes with existing one to one negotiation strategy (Fudzee, 2011) that is most works dealing with client-side quality evaluation perform recommendations during service selection process. In this strategy, the waiting time has been focusing to reflect the response time. The broker reaches the requested service by searching the priority function. The current server load checks by SLA assessor and estimates the value of request it can be served within offered waiting time.

This strategy offered single session task by SLA assessor to be consider by waiting time and at the same time the new waiting time for the request that might be rejected. Figure 2 depicted an example of implementing E(W) QoS negotiation between brokers with a content adaptation provider.

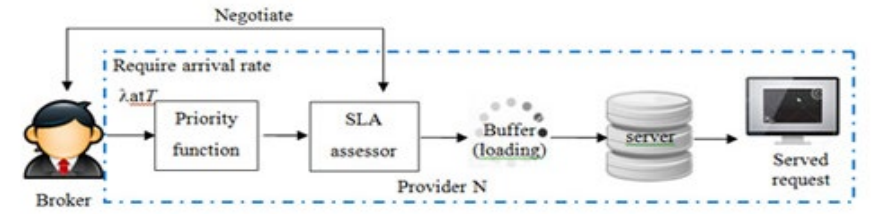

Figure 2: One-to-one negotiation

From the Figure 2, once the agreements are entered into, it becomes necessary to monitor their conditions, which will commonly relate to timeliness, reliability and request throughput, at run time (Raimondi, Skene \& Emmerich, 2008). The problem with this strategy is broker should have to stick around for a few seconds to receive the adapted content. As a result, there will be a heavy load of incoming request, false of information and disclaimed information. To overcome this problem, we propose a strategy for service providers to evaluate incoming requests and capable of offering the new response time. The proposed strategy called One-to-Multiple negotiation. This strategy takes into account the current server load and enables a mechanism for the user to evaluate whether the new response time can be accepted or not.

\section{General MCDM Design Model and Methodology for Teaching Performance Evaluation}

This section, we will describe about the proposed of One-to-Multiple Negotiation strategy that has capability to determine that SLA can be settled with overall waiting time for all task rather than a single task. This improvement strategy based from (Al-Jaljouli \& Abawajy, 2012) will consider less of response time by applying overall task to a certain request. Figure 3 illustrates the one-to-multiple negotiation strategy.

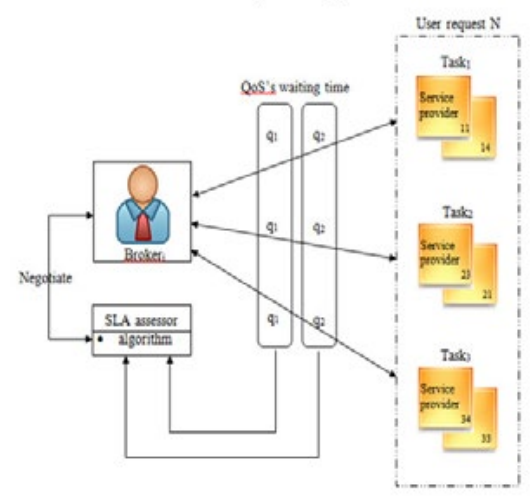

Figure 3: One-to-multiple Negotiation strategy

Assuming the brokeri request several tasks namely task1, task2 and task3. Next, the SLA waiting time (QoS) will be considered as 3 seconds. The service provider will check the current load and calculating the request that can be served. Further assume the service provider will be served by two options. The first option in task 1 , task 2 and task 3 offered exactly 3 seconds and the second option will offer 3 seconds in task 1,2 seconds in task 2 and 4 seconds in task3. The estimation of QoS waiting time describes thru Table 1.

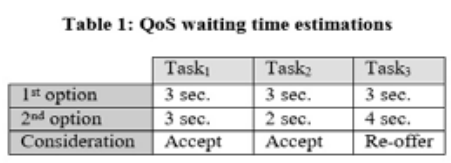

These tasks will accept all the requested needs from the service provider by replying back any feedback within the two options given. All tasks will take maximum of 3 seconds per services to fulfill the settlement of SLA. Typically, all the tasks completed in terms of technically. Therefore, this strategy can allow user to get any responds from several task and it will decrease user's perception about waiting time. By applying this method, the enhance user experience of the content adapted and also provide flexible and scalable service-based content delivery mechanism. An algorithm is applied to this strategy at SLA assessor for improvement settlement rate of waiting time factor considers by QoS. Table 2 list the common symbol used for this paper based on proposed model.

Table 2: common symbol

\begin{tabular}{|l|l|}
\hline Symbol & Description \\
\hline$N$ & Arrival rate of request \\
\hline$E(W)$ & Expected waiting time \\
\hline$S$ & Server load \\
\hline
\end{tabular}

\section{SLA Assessor}

The inputs for the enhancement of SLA assessor algorithm pioneered from Liu et. al, (2010) are the requests inclusive the current server load, the QoS and their priorities. Figure 4 states the algorithm by communication steps of settlement SLA by SLA assessor.

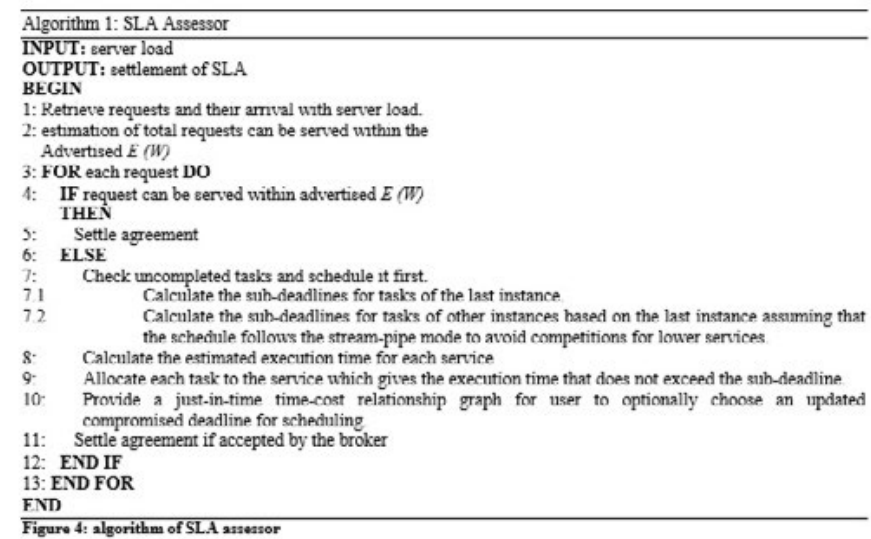

Speciffically, during the QoS negotiation process, the waiting time QoS E (W) of each content adaptation server will be evaluated by manipulating Pollacrek-Khintchine formula as in (Pathan, \& Buyya, 20019): $E / W=2 E\left(X^{3}\right) / 2(l-s) \quad \mathrm{F}_{\mathrm{q}}(1)$

Next, E(X) clarifies as the mean service time. A Bounded Pareto adopted from (Pathan and Buyya. 2009) allocation followed of each incoming request size. A Bounded Pareto B $(k, p, \alpha)$ of probability density function (Pathan, \& Buyya, 2009) is:-

$$
f(x)=\frac{a x^{*}}{1-\frac{d}{2} x^{2}} x^{-\alpha-1}
$$

$\mathrm{E}_{\text {q. (2) }}$

where the task size variation represents by $\alpha, \mathrm{k}$ and $\mathrm{s}$ which are smaller and the largest possible task size with $(\mathrm{k} \leq \mathrm{x} \leq \mathrm{s})$. High variability is observed when ( $\alpha$ approx 1$)$ and when ( $\alpha$ approx 2 ) observed by moderate variability. The expected waiting time of $\mathrm{E}(\mathrm{W})$ is predict before a request being serviced so that the requests potentially being rejected by offering new service time of $\mathrm{x} . \mathrm{E}(\mathrm{X})$ of the mean service time issues by the following mathematical formula (Pathan, \& Buyya, 2009):

$$
E\left(X^{j}\right)= \begin{cases}\frac{\left.\alpha^{\prime}\left(\frac{k}{s}\right)^{\alpha}-\left(\frac{k}{s}\right)^{j}\right)}{(j-\alpha)\left(1-\left(\frac{k}{s}\right)^{\alpha}\right)} & \text { if } j \neq \alpha \\ \frac{\alpha k^{\alpha} \ln \left(\frac{s}{k}\right)}{\left(1-\left(\frac{k}{s}\right)^{\alpha}\right)} & \text { if } j=\alpha\end{cases}
$$

The current load estimations within given number of the requests by plotting E(W). For instance, the waiting time of QoS had been advertised by three service provider namely task_1, task_2 and task_3 by assuming the fair load of server (0.6) and the $\mathrm{N}$ as 50 . The $\mathrm{E}(\mathrm{W})$ estimated by SLA assessor and only form 30 to 80 requests can be realized to be served by $\mathrm{E}(\mathrm{W})$ being offered. As a result, the SLA is settled with the first 30 until 70 of requests by task_1 of the overall task. 


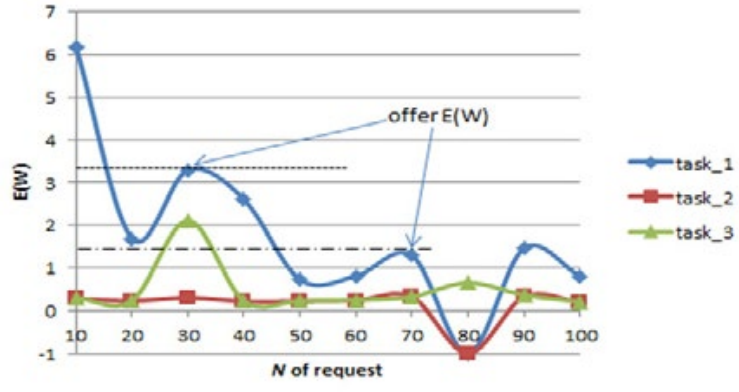

Figure 5: offered $E(W)$ against three tasks based on current load.

From the Figure 5, assuming that the E(W) evaluates by SLA assessor for the rest of requests. Further assume that the first of 30 requests is offered $3.3 \times 109$ for the $\mathrm{E}(\mathrm{W})$, then the rest of 70 requests offered with $1.5 \times 109$. The SLAs are settled if the broker accepts the E(W) by negotiating at task_1 otherwise the task_2 and task_3 will continuing the journey to be received the requests. From this, we can conclude that the overall task can be agreed by both of the broker and service provider referring to the service level agreement.

\section{Conclusion}

We use the simulation to study the efficiency of the service provider selection by focusing on the expected waiting time of $\mathrm{E}(\mathrm{W})$ by takes into account the current server load and enables a mechanism for the user to evaluate whether the new response time can be accepted or not.

To improve the performance of expectation waiting time, the current methodology from (Md Fudzee, \& Abawajy, 2011) has been followed. Table 3 shows the simulation setting and the parameter to this study.

\begin{tabular}{|l|l|}
\multicolumn{2}{|c}{ Table 3: Simulation settings } \\
\begin{tabular}{|l|l|}
\hline Parameter & Value \\
\hline$\lambda$ & 50 to 100 \\
\hline$\alpha$ & 2 \\
\hline$\rho$ & 0.5 (lightly load) to 0.9 (heavy load) \\
\hline$k$ & 1010.15 \\
\hline$s$ & $10^{10}$ \\
\hline $\begin{array}{l}\text { service } \\
\text { distribution }\end{array}$ & $\begin{array}{l}f(x)=\alpha k^{\alpha} \\
1-\alpha-1\end{array} \quad(-) \quad(k \leq x \leq s)$ \\
\hline $\begin{array}{l}\text { E(W) } \\
\text { acceptance } \\
\text { rate }\end{array}$ & $0 \%$ to $30 \%$ \\
\hline
\end{tabular} \\
\hline
\end{tabular}

The improvement approach based from (Zheng, Zhang, \& Lyu, 2010) and (Zhang, Zheng, \& Lyu, 2011) gives more implication to the SLA. We analyzed the performance of the proposed strategy in terms of SLA settlement under various conditions. The results indicate that the proposed strategy performs well.

Referring to the Figure 6, the SLA acceptance rate shows at y-axis and the function of the number requested show by $\mathrm{x}$-axis. We transforms $\mathrm{N}$ of incoming request from 50 to 100 from the simulation settings. The server load in this case takes into account as 60 by a fair load of 0.6 advertised by $\mathrm{E}(\mathrm{W})$. The acceptance rate of QoS offered by $\mathrm{E}(\mathrm{W})$ spread of $0 \%$ to $30 \%$.

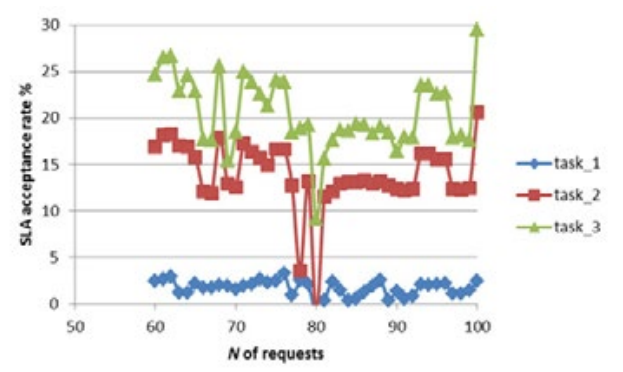

Figure 7: SLA acceptance rate versus requested numbers (default server load 0.6)

As in Figure 6, there are three task calls for a request, task_1, task_2 and task_3. From the server load at 60, the three tasks play role to give their service with difference number. There are offered the dramatically lowest request from task_1 while the other two tasks (task_2 and task_3) offer considerably higher chances to run the request along at $\mathrm{x}$-axis. The requested along $\mathrm{x}$-axis increases about $23 \%$ at task_3 then at task_1. This simulation proves that task_3 can served with higher cumulative rate of $E(W)$. As a result, the settlement rate will be more flexible due to the higher cumulative of $\mathrm{E}(\mathrm{W})$. The expectation for the simulation is when the settlement rate is higher so that the SLA is settled refer to the agreement. This is because the strategy can reduce the upcoming request from second option for each task once the first option cannot satisfy with advertised waiting time.

\section{Conclusion}

Content adaptation is a potential solution for tailoring multimedia web content according to the users' preferences and heterogeneous devices' constraints. Content adaptation can be done as third party service over the Internet. User consumed that the adaptation service can pay them good service. The quality should include the human psychological factors. One of these factors is the maximum time a user can wait for the output to be displayed. Thus, response time is one of the qualities of service (QoS) to be considered in assessing the deliverability of content adaptation services. Practically, the service provider should able to determine a current deliverable response time before the service level agreement (SLA) is settled with the users. We summarized our contribution into: (1) we proposed the improvement of SLA assessor tailor to the SOCA framework, (2) the proposed strategy for SOCA framework simulated by several tasks. In future, we plan to study on monitoring of the strategy by serve a given task advertised of QoS.

\section{References}

Al-Jaljouli, R., \& Abawajy, J. (2012). Strategies for Agent-Based Negotiation in E-Trade. Network and traffic engineering in emerging distributed computing applications, 43-65.

Arai, K., \& Tolle, H. (2010). Automatic e-comic content adaptation. International Journal of Ubiquitous Computing, 1(1), 1-11.

Carreras, A., et. al., 2009. Context-aware and DRM-enabled content adaptation platform for collaboration applications. IEEE Multimedia.

Egger, S., Hossfeld, T., Schatz, R., \& Fiedler, M. (2012, July). Waiting times in quality of experience for web based services. In Quality of Multimedia Experience (QoMEX), 2012 Fourth International Workshop on (pp. 86-96). IEEE.

Fudzee, M. F. M., Fudzee, M. F. M., \& Abawajy, J. H. (2011). A Strategy of Negotiating QoS for Content Adaptation Services. Deakin University, School of Information Technology.

Fudzee, M. F. M., Abawajy, J., \& Deris, M. M. (2010, May). Multi-criteria content adaptation service selection broker. In Cluster, Cloud and Grid Computing (CCGrid), 2010 10th IEEE/ACM International Conference on (pp. 721-726). IEEE.

Kosinski, M., Stillwell, D., \& Graepel, T. (2013). Private traits and attributes are predictable from digital records of human behavior. Proceedings of the National Academy of Sciences, 110(15), 5802-5805.

Liu, K., Jin, H., Chen, J., Liu, X., Yuan, D., \& Yang, Y. (2010). A compromisedtime-cost scheduling algorithm in swindew-c for instance-intensive costconstrained workflows on a cloud computing platform. International Journal of High Performance Computing Applications, 24(4), 445-456.

Lobo, D., Kaskaloglu, K., Kim, C. Y., \& Herbert, S. (2011). Web usability guidelines for smartphones: A synergic approach. International journal of information and electronics engineering, 1(1), 33-37.

M.F. Md Fudzee \& J. Abawajy. 2011. QoS-based content adaptation service selection broker. Elsevier's Future Generation Computer System 27 (3), 256-264.

Malandrino, D., Mazzoni, F., Riboni, D., Bettini, C., Colajanni, M., \& Scarano, V. (2010). MIMOSA: context-aware adaptation for ubiquitous web access. Personal and Ubiquitous Computing, 14(4), 301-320.

Pathan, M., \& Buyya, R. (2009). Architecture and performance models for QoS-driven effective peering of content delivery networks. Multiagent and Grid Systems, 5(2), 165-195.

Raimondi, F., Skene, J., \& Emmerich, W., 2008. Efficient online monitoring of web-service SLAs. In Proceedings of the 16th ACM SIGSOFT International Symposium on Foundations of software engineering (pp. 170-180). ACM.

S.J.H. Yang \& N.W.Y Shao. 2007. Enhancing pervasive Web accessibility with rule-based adaptation strategy. Journal of Expert Systems with Applications, 32, 1154-1167.

Yilei Zhang, Zibin Zheng, and Michael R. Lyu, (2011) Exploring Latent Features for Memory-Based QoS Prediction in Cloud Computing, in Proceedings of the 30th IEEE Symposium on Reliable Distributed Systems (SRDS 2011), Madrid, Spain, Oct.4-7, 2011.

Zibin Zheng, Yilei Zhang, and Michael R. Lyu, 2010. Distributed QoS Evaluation for Real-World Web Services, in Proceedings of the 8th International Conference on Web Services (ICWS2010), Miami, Florida, USA, July 5-10, 2010, pp.83-90. 